\section{Pancreatic-Pleural Fistula Demonstrated by Endoscopic Retrograde Cholangiopancreatography}

Acute pancreatitis is a common emergency in surgical wards. In its aggressive form, it can involve intraperitoneal and retroperitoneal organs with inflammation (1). In $5-15 \%$ of cases, acute pancreatitis is associated with necrotic inflammation, abscess, or pseudocyst formation. Chest complications are present in $1-17 \%$ of these cases, leading to impaired pulmonary function, low arterial $\mathrm{PO}_{2}$, and pleural effusion (2).

A 46-year-old woman was admitted to the internal department of our hospital due to bilateral subcostal pain and dyspnea. She had a history of three episodes of acute pancreatitis. The serum and urine amylase levels were elevated (1.371 and 9.340 IU/I), demonstrating another incident of acute pancreatitis. The chest radiograph showed a massive left pleural effusion (Figure 1). Repeated puncture of the thoracic fluid was carried out; the amylase level in the fluid was $70,000 \mathrm{IU} /$. The presence of a pseudocyst with a diameter of $15 \mathrm{~mm}$ in the pancreatic body was verified by a CT scan. The patient's symptoms declined with conservative treatment, but the thoracic effusion refilled, and it was therefore decided to carry out surgical treatment. Before surgery, an endoscopic retrograde cholangiopancreatography (ERCP) examination was carried out, demonstrating the pseudocyst and a wide pancreatic-pleural fistula (Figure 2). The surgical solution was a distal pancreatic resection with splenectomy and cholecystectomy. The patient recovered without any complications, and left the hospital on the 20th postoperative day.

Pancreatic-pleural fistula is a rare finding, and pleural effusion due to direct communication between the pancreatic pseudocyst and the chest is unusual (2). The fistula can lead through a natural hiatus into the mediastinum, or by direct penetration through the dome of the diaphragm (3). Abdominal symptoms are not always present (1). Visualization of the fistula is possible with ERCP, but CT and US are also advisable (4). The treatment can be started conservatively, but if the result is not adequate within two weeks. surgical treatment is mandatory, and this usually means pancreatic resection (5). Delaying surgical treatment can result in high morbidity and mortality (4).

P. Ondrejka', F. Siket ', I. Sugár', J. Faller '

' Dept. of Surgery, Semmelweis University Medical School, Budapest, Hungary;

${ }^{2}$ Fourth Internal Dept., St. John's Hospital, Budapest, Hungary

\section{References}

1. Fielding FM, Rossi RC, Munson JL, Remine SG, Braasch JW. Acute pancreatitis and pancreatic fistula formation. $\mathrm{Br} J$ Surg 1989; 76: 1126-8.

2. Frey CF. Pancreatic pseudocyst: operative strategy. Ann Surg 1978; 188: 652-62.

3. Kaye MD. Pleuropulmonary complications of pancreatitis. Thorax 1968; 23: 297-306.

4. Burges NA, Moore HE. Williams JO, Lewis MH. A review of pancreatic-pleural fistula in pancreatitis and its management. HPB Surg 1992; 5: 79-86

5. Kohler H, Schafmayer A, Lepsien G, Peiper HJ. Pancreatikopleurale Fisteln. Beitrag zur Klinik und Therapie eines seltenen Krankheitsbildes. Chirurg 1990; 61: 639-42.
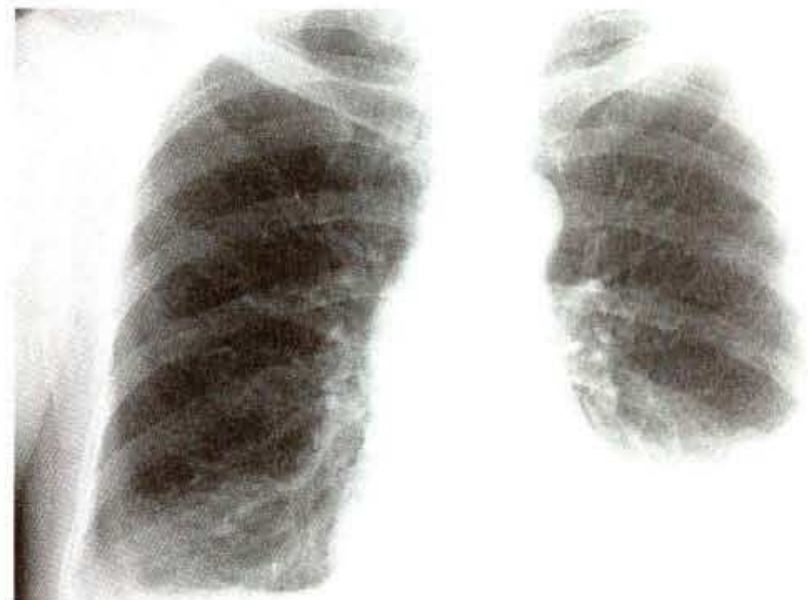

Figure 1: Chest radiograph, showing the left pleural effusion reaching the eighth costal line.

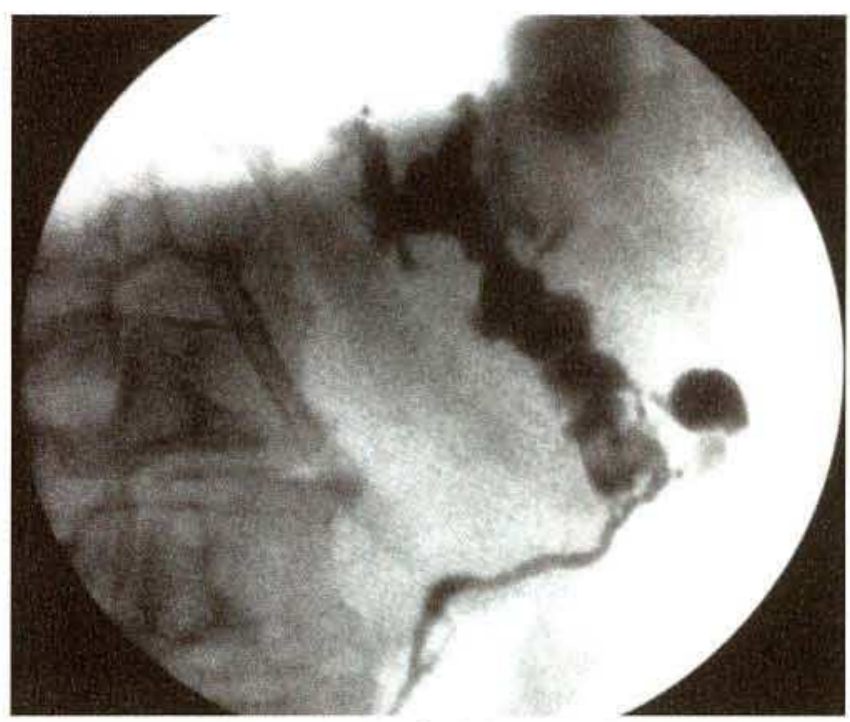

Figure 2: ERCP demonstrating the fistula between the pancreatic pseudocyst and the left thoracic cavity

Corresponding Author

P. Ondrejka, M.D., Ph. D.

Dept. of Surgery

Semmelweis University Medical School

Diosarok u. 1

1125 Budapest XII

Hungary

Fax:+36-1-1 56-3049 